\title{
Intelligent Assessment, Diagnosis and Planning of All- age Friendly Communities Based on Random Forest
}

\author{
Geqi Luo, School of Geographic Science, Hunan Normal University, Changsha 410081, P.R.China \\ Chao Liang, School of Geographic Science, Hunan Normal University, Changsha 410081, P.R.China \\ Junlin Huang*, School of Geographic Science, Hunan Key Laboratory of Geospatial Big Data Mining and \\ Application, Hunan Normal University, Changsha 410081, P.R.China
}

(Fund Project: National Natural Science Foundation of China(52008167,41871318); National Students' Platform for Innovation and Entrepreneurship Training Program(202010542015))

\begin{abstract}
Based on the random forest algorithm model, this paper constructs a set of intelligent evaluation, diagnosis and planning methods for all-age friendly communities to support the formulation of planning governance policies and planning actions for accurate allocation of facilities, and takes Changsha as an example. (1) From the evaluation results of friendliness impact factors, public leisure space, safety and health are the key impact factors of "public friendliness", the sound status of community services is the key impact factor of "youth friendliness", the demand for culture and entertainment is the key impact factor of "children and the elderly friendliness", and the degree of public friendliness construction is the key impact factor of all age friendly communities, but the overall difference of the factors is small; (2) According to the diagnosis results based on RF, each kindness degree of Changsha community presents an obvious "core-periphery "pattern, and the degree of friendliness is highly correlated with the development degree of the area; (3) Through the comparison of the original regulatory planning and model planning results, it is found that the intelligent model based on RF and multi-agent can accurately identify the short board of community development, and put forward the planning improvement scheme.
\end{abstract}

\section{Keywords}

Random Forest; All-age friendly; Intellectualization; Community governance; Changsha City

\section{Introduction}

Community is not only the "cell" of urban "organic life", but also the smallest unit of national governance system. The "Friendliness" experience of all age groups has become a key indicator to highlight the level of governance modernization. Related theories, technical standards and policies have become the focus of theoretical research and practical exploration. The continuous emergence of urban construction models from "liveable city"1 to "suitable for aging"2,3 and "child friendly"4 reflects the continuous upgrading of the world's response to the needs of people of all ages ${ }^{5}$, such as liveable, suitable for work, suitable for travel, suitable for study and suitable for maintenance, so as to promote the implementation of the concept of friendship between children, youth ${ }^{6,7}$ and the elderly ${ }^{8,9,10}$ in urban construction and management. Since 2000, based on the emerging problems in urban construction and development, a "all-age-friendly community" characterized by intergenerational integration has been put forward to meet the requirements of the new era ${ }^{11,12,13}$. In 2010 , the United Nations first proposed the "inclusive city" to realize 
the sustainable urban development and construction of the whole life cycle from children, youth to the elderly from the three dimensions of sharing, participation and integration, which has been responded by many countries, including China ${ }^{14,15,16}$. Canada proposed the "all-age-friendly city" project in 2014, and China proposed it in 2020 "All-age-friendly and inclusive society construction project", China's Xiong'an new area has also put forward the goal of building a "all-age-friendly city".

As a whole, the current research on All-age friendly and inclusive cities has the following characteristics: (1) most of the urban evaluation research on the community scale is the liveability evaluation of the facility dimension ${ }^{17,18}$, while there are relatively few studies ${ }^{19}$ on the specific needs of residents of different ages for diverse survival and life; (2) The research object scale mainly focuses on the administrative area category at the street level, while the community scale research is less ${ }^{20}$. The index system based on the humanistic perspective and the qualitative theory of existing community rule indicators need to be broken through ${ }^{21}$; (3) In terms of community evaluation methods ${ }^{22}$, the data fusion and methods of analysis and evaluation of all age friendly community construction indicators based on urban multi-source data and machine learning algorithms need to be promoted; (4) It is suggested to identify timeliness and guiding significance of the key factors affecting community construction ${ }^{23}$, and break through it in the face of the changing real society.

Random forest (RF) is a machine learning algorithm proposed by Leo ${ }^{24}$ for combinatorial classification (Classification and Regression Tree, CRAT). It has strong data mining ability and high accuracy on prediction in multivariable data processing, and has excellent tolerance to outliers and noise, which can achieve high scores with optimal parameters and minimum error according to limited training samples, and establish a weight learning mechanism among multiple variables, so as to solve the "over-fitting" problem ${ }^{25}$ of an attribute evaluation in complex and nonlinear large-scale systems. In addition, All-age friendliness is a complex system involving the interaction of facility allocation, socio-economic and personal behaviour and feelings. Its evaluation measurement system has complexity, non-structural and random uncertainty. More robust and flexible measurement methods are needed to deal with nonlinear relationships, high-order correlations and even missing values. At the same time, with the passage of time and space, the impact of different indicators on friendliness may change, and the initial weight may not be in line with the actual situation, which further promotes the development of the measurement model to non-parameterization. As a nonparametric tree model, RF has all the advantages of the previous calculation methods of composite index system, and has better performance in the processing of multivariable nonlinear relationship and weight dynamics. It can prevent the reduction of accuracy caused by noise and data loss in training samples. In theory, RF is an ideal tool to evaluate the age-friendly situation.

\section{2 Construction of RF Model for age friendliness measurement}

\subsection{Basic principles}

The core of constructing the RF model of community age friendliness measurement is to reveal the corresponding rules between each index and the measurement results, which is undertaken by the classification tree. The classification tree is composed of root node, child node and leaf node three parts. Among them, the root node represents the observation value of the index variable, the leaf node represents the linear decomposition of the index variable, the path from the root node to the leaf node corresponds to the decomposition rules, the leaf is the node without the next branch, and the growth process of the classification tree represents the training process of the sample set. The basic principle of $\mathrm{RF}^{26}$ is shown in Figure 1 Firstly, a classifier is used to create a "forest", and the data is randomly selected through the bootstrap method to grow into a classification tree groups. Each tree will grow completely without being built. When generating the tree, select the observation data of each index variable through 
the Bootstrap method to create a differentiated and randomized training sample set, and input the training data set into the classification tree to form a classifier, using the Random Forest law to split each node, and decompose the values of each index variable with nonlinear relationship with the evaluation object into potential leaves with linear relationship, The result with the most votes is returned as the indicator decomposition result; Secondly, the distribution structure of leaves is analysed to extract and calculate the contribution value (weight) of each decomposition index variable to the evaluation results. The decomposition index weights are formed into a one-to-one corresponding relationship set between index variables and weights according to the linear mapping rules of classification tree, and the RF model is output, The average value of the comprehensive weighting of the RF characteristic value of the index variable and its corresponding weight value on each leaf node is the calculated result.

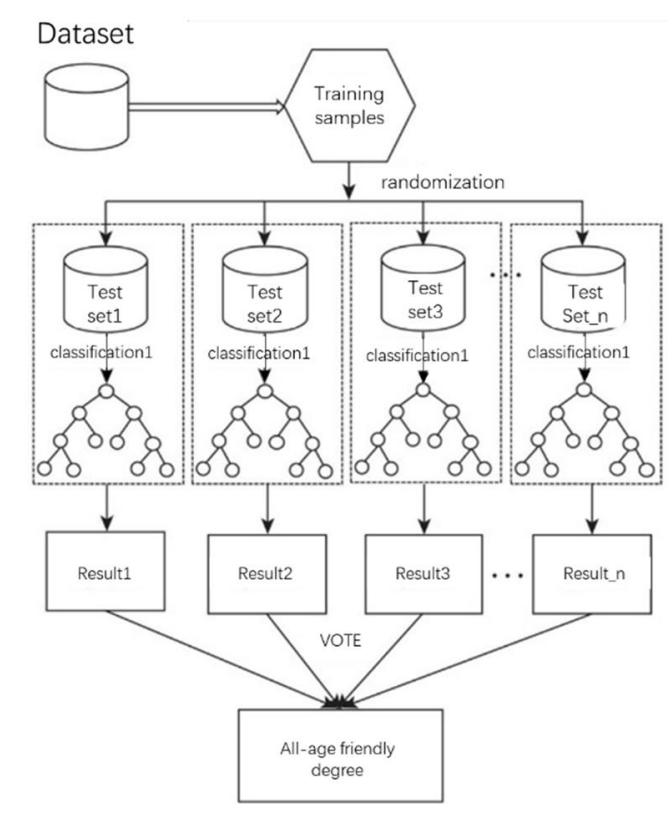

Figure 1 Basic principle of Random Forest model.

\subsection{Model Construction}

This study constructs the model through three steps.

\section{Step one: Construct a classification tree.}

Two very important hyper-parameters need to be present in the specific implementation of RF, the number of classification trees $(\mathrm{k})$ and the optimal node splitting times $(\mathrm{m})$ when each classification tree is constructed. The former determines the overall size of the Random Forest, and the latter determines the status of a single classification tree. These two parameters must be optimized to improve the accuracy of the model in data processing. The specific process of constructing a classification tree model containing $\mathrm{K}$ All-age friendly evaluation trees is as follows Figure 2:

(1) Get training set. The original training sample set of age friendliness evaluation is recorded as $T=\{(x 1$, $\left.y 1),(x 2, y 2), \ldots,\left(x_{m}, y_{m}\right)\right\}$. Bootstrap sampling method is used to form a random vector sequence Tt from two-thirds of the samples in $T$, which is repeated $K$ times to form $K$ independent and identically distributed training sets $\{T k, t=1,2, \ldots, k\}$ 
(2) Randomly select the node characteristic index. For $k$ data sets, establish the deepest classification tree without pruned processing. By calculating the Gini index contained in each index variable, the Gini index of node $n$ is defined as:

$$
\operatorname{Gini}(n)=\sum_{i \neq j} p\left(w_{i}\right) p\left(w_{j}\right)=1-p^{2}\left(w_{j}\right)
$$

Where $\boldsymbol{p}\left(\boldsymbol{w}_{\boldsymbol{i}}\right)$ is the frequency of the number of samples belonging to class $i$ in the number of training samples on the $\mathrm{n}^{\text {th }}$ node.

(3) Determine the split node. Split the candidate attribute with the largest Gini index and recalculate the Gini index. Repeat the splitting step until the Gini index is less than the predetermined threshold, and finally form a decision-making "forest" with $\mathrm{K}$ trees.

(4) Recursive classification. Feedback the decision result of the number of $K$ trees, and determine the best linear decomposition mode of the index variable according to the principle of the largest number of votes.

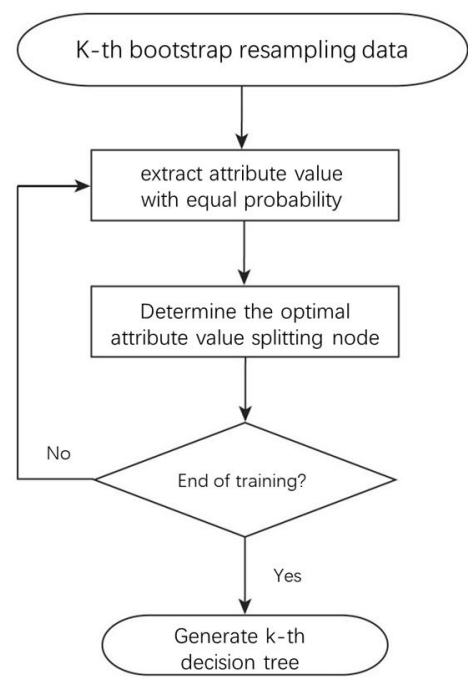

Figure 2 Classification tree construction

\section{Step two: Calculate the weight of RF.}

Random Forest increases the difference between classification models by constructing different training sets, so as to improve the extrapolation prediction ability of combined classification models. In the process of decision tree classification, only part of the data of the original data set is used, and the remaining data does not play a role. In order to avoid over-fitting problem, RF uses the Gini value method to calculate the importance of each decomposition index based on this part of out of bag data (OOB). The specific implementation steps mainly include: assuming that the Gini index of the original segmentation of the I classification tree is Gini $\boldsymbol{e}_{\boldsymbol{i}}$, and change the attribute value $\mathrm{x}$ of the $\mathrm{j}$ decomposition variable of $\mathrm{OOB}$ by random sequence, and recalculate the new Gini index as Gini $e_{i}^{j}$, then the weight of attribute $\mathrm{X}$ in the corresponding single classification tree is expressed as Gini $\boldsymbol{e}_{\boldsymbol{i}^{-}} \mathbf{G i n i} \boldsymbol{e}_{\boldsymbol{i}^{j}}^{j}$, the dynamic weight RFW of the $j$ decomposition index is the mean value of Gini index of all classification trees in the forest, and the calculation formula is as follows: 


$$
\begin{gathered}
\Delta_{j}=\left|\sum_{j=1}^{N}\left(\operatorname{Gini}_{e_{i}}-\operatorname{Gini}_{e_{i}^{j}}\right) / N\right| \\
R F W_{j}=\Delta_{j} / \sum_{j=1}^{N} \Delta_{j}
\end{gathered}
$$

Where: $\Delta_{j}$ is the decrease of Gini index; $\mathrm{N}$ is the number of indicators; $\boldsymbol{R} \boldsymbol{F} \boldsymbol{W}_{\boldsymbol{j}}$ is the weight value of the $\mathrm{j}$ decomposition index, which meets the following requirements: $\sum_{j=1}^{N} R F W_{j}=1$

\section{Step three: Weighted combination.}

Firstly, the RF weight of each decomposition variable is output, and the optimal simulation relationship between each index variable and the response variable (community All-age friendliness) is generated according to the linear mapping rule of classification tree; Then input the sample set to be tested into each trained RF classification tree. The comprehensive weighting of the eigenvalue and its weight value on each leaf node is the community All-age friendliness level of the tree. Finally, the average value of community All-age friendliness on the leaf node of each classification tree is the calculated community All-age friendliness value.

\section{Step four: Fuse method.}

Considering the shortcomings of site selection in the All-age friendly planning model based on Random Forest, previous studies ${ }^{27}$ have used multi-agent and other methods for intelligent site selection. This paper integrates it into the whole model process to explore a set of intelligent community planning model based on the whole technology system of Random Forest, which is specifically divided into:

(1) Agent of Citizen demand level:

$$
D=\frac{d_{i}^{+}}{\left(d_{i}^{+}+d_{i}^{-}\right)}
$$

Where, $\boldsymbol{d}_{i}^{+}, \boldsymbol{d}_{i}^{-}$is the distance between each index point and the best and worst index point of the sample; The value range of $D$ is $[0,1]$. The smaller $D$ means that the length difference between the target bucket and the longest bucket is smaller, and the bucket is more about balanced; Establish the criteria for short board elements, and the equilibrium degrees are $[0,0.3],(0.3,0.6],(0.6,1.0]$, corresponding to dominant elements, restrictive elements and short board elements respectively.

(2) Agent of population flow:

$$
D\left(x_{i}, y_{i}\right)=\frac{1}{u r} \sum_{i=1}^{u} k\left(\frac{d}{r}\right)
$$

Where $\boldsymbol{D}\left(\boldsymbol{x}_{\boldsymbol{i}}, \boldsymbol{y}_{\boldsymbol{i}}\right)$ is the Kernel density estimation at the spatial position $\left(\boldsymbol{x}_{\boldsymbol{i}}, \boldsymbol{y}_{\boldsymbol{i}}\right) ; \boldsymbol{r}$ is the distance attenuation threshold; $u$ is the number of feature points whose distance from the position $\left(\boldsymbol{x}_{\boldsymbol{i}}, \boldsymbol{y}_{\boldsymbol{i}}\right)$ is less than or equal to $r$; Function $\mathrm{k}$ represents spatial weight function; $\mathrm{d}$ represents the Euclidean distance between the current feature point and two points $\left(\boldsymbol{x}_{\boldsymbol{i}}, \boldsymbol{y}_{\boldsymbol{i}}\right)$. 
(3) Agent of government:

The government agent will make a comprehensive decision according to the short board level within the community, the activity of the traffic agent in space and the location of the spatial location that the citizens intend to build. The specific decision table is shown in Table 1.

Table 1 Multi agent location decision table

\begin{tabular}{ccccccccccc}
\hline $\begin{array}{c}\text { Agent of Citizen } \\
\text { demand level }\end{array}$ & \multicolumn{1}{c}{ High } & & Medium & & Low \\
\hline $\begin{array}{c}\text { Agent of } \\
\text { population flow }\end{array}$ & High & Medium & Low & High & Medium & Low & High & Medium \\
\hline $\begin{array}{c}\text { Agent of } \\
\text { government }\end{array}$ & $\mathrm{V}$ & $\mathrm{V}$ & $\mathrm{x}$ & $\mathrm{V}$ & $\mathrm{V}$ & $\times$ & $\times$ & $\times$ \\
\hline
\end{tabular}

\subsection{Research Process}

Taking the community as the governance unit, this study introduces the concept of All-age friendliness and algorithms such as Random Forest, bucket theory and multi-agent to explore the theory of community construction in the whole life cycle and optimize the evaluation index system, multi-source data mining for age-based integration of community construction, as well as the innovation of space analysis methods The construction of community planning governance method with the coupling of All-age friendly evaluation and planning intervention mechanism aims to solve the traditional research limitations such as research scale, technology integration, renewal and optimization, model integration and so on. By constructing the algorithm model based on Random Forest, the "cell physical examination" of the whole age friendly community is carried out, supplemented by basic algorithms such as barrel theory, so as to realize the community dynamic evaluation feedback mechanism based on intelligent algorithm, and then explore the realization and optimization path of the whole age, the whole people and the whole age friendly and inclusive, take people-oriented and the whole age friendly and inclusive as the research concept, and take the community as the unit to create the residents' life "City of life".

The All-age friendly community intelligent assessment, diagnosis and planning process is mainly divided into three steps: overall assessment, problem diagnosis and planning exploration. The specific process is shown in Figure 3:

1) Multi source data fusion. Based on the existing data, the data can be roughly divided into four modules: basic geographic data, spatial planning data, socio-economic data and urban operation data, so as to integrate multi-source data and establish database.

2) Key factor identification. According to the dimension setting of community assessment planning system and based on Random Forest model, the planning key factors of index systematization data are identified, and the planning effective factors are extracted.

3) Model intelligent evaluation and problem diagnosis. Based on the Random Forest, the community in the study area is evaluated, and the current situation and problems of the construction area are analysed. The logical thinking of intelligent evaluation model is divided into data processing, training model and model evaluation.

4) Governance model construction. The construction of governance model will be deconstructed and re systematized from the direction of multiple actors, which is highly targeted for the construction of a single community. The participants in community planning and governance are mainly divided into residential subjects, decision-making subjects, construction subjects and supervision subjects, which carry out index system construction, intelligent evaluation, planning intervention and operation supervision. 


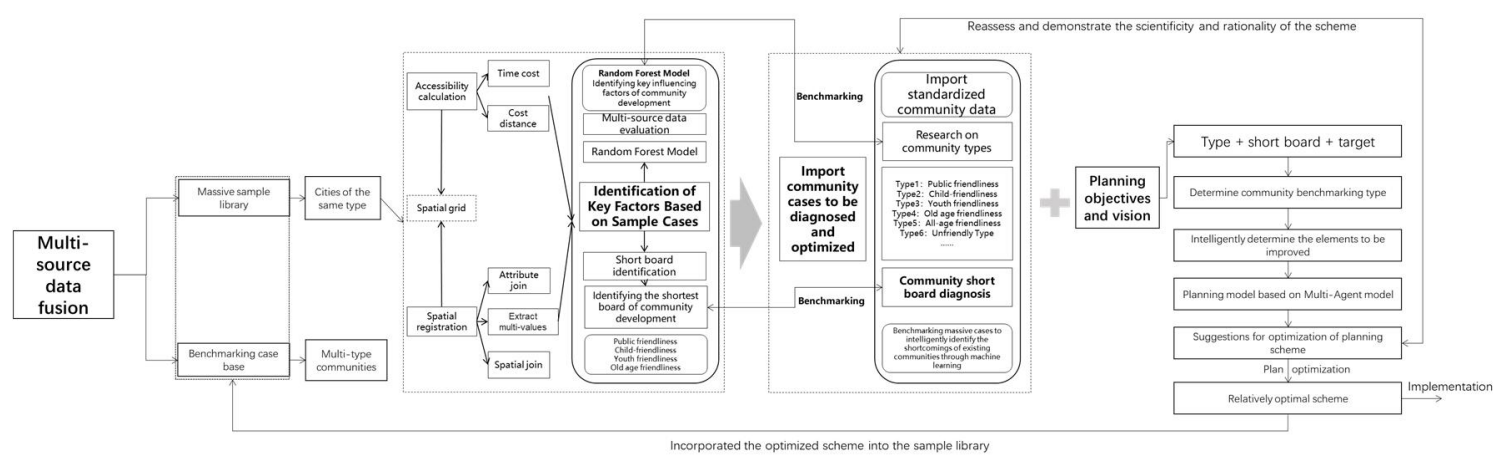

Figure 3 All-age friendly community assessment, diagnosis and planning research process

\section{Performance Analysis}

Six districts and one county in Changsha are the main gathering places for urban personnel, with relatively perfect facilities construction. It is the most concentrated area for community renewal and construction in Changsha, so its community construction is highly representative. The study covers 966 basic communities in Furong district, Kaifu District, Yuelu District, Yuhua District, Tianxin District, Wangcheng district and Changsha County.

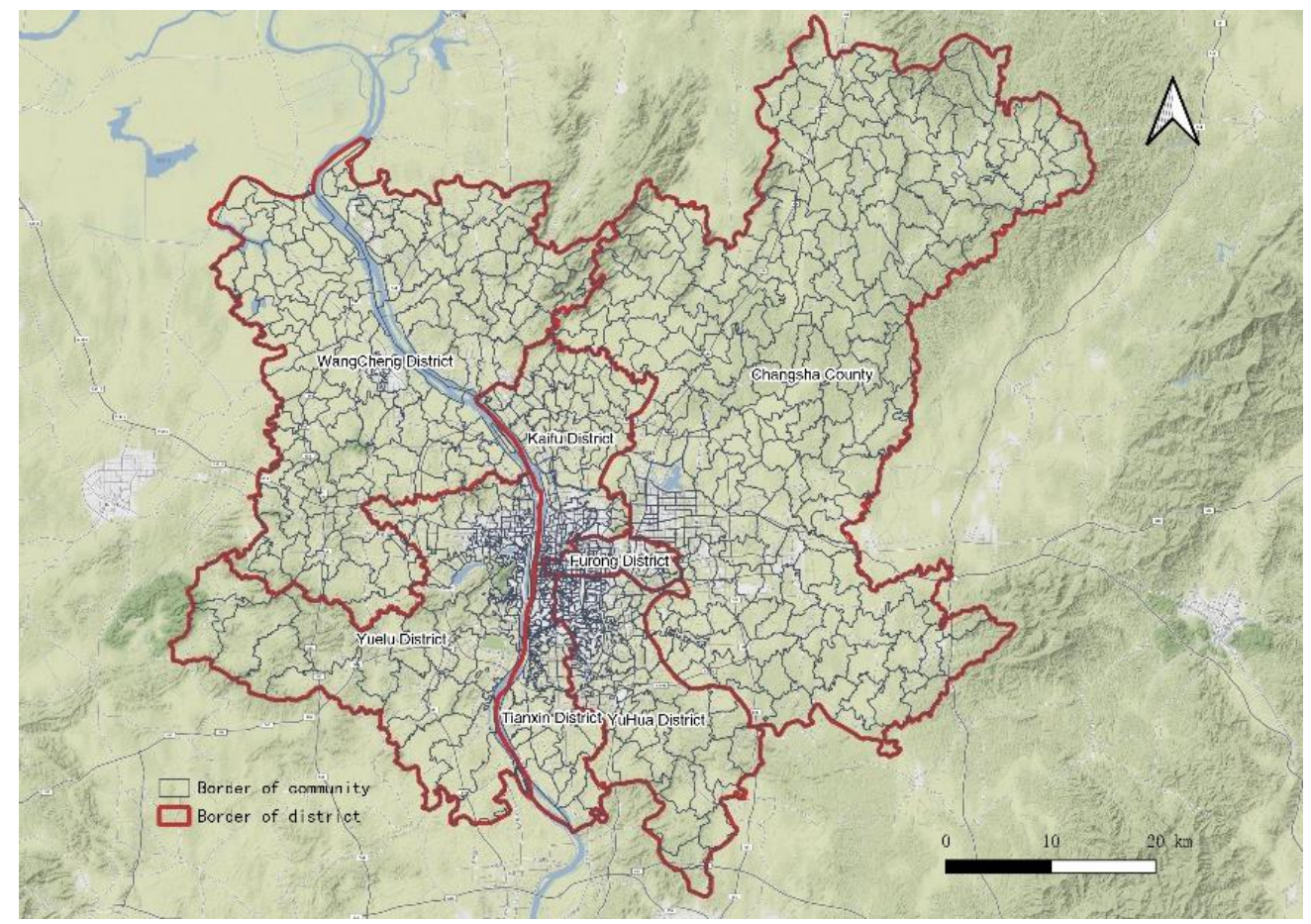

Figure 4 Overview of the study area

\subsection{Overall assessment: classification based on identification of key influence factors}

Feature engineering is the most important part of machine learning. How to construct features determines the upper limit of machine learning. The construction of index system in this paper consists of index sea selection, collinearity test and manual evaluation. Firstly, a comprehensive index system database is formed by referring to the more mature urban physical examination index system and livable city index system; Secondly, by studying the selection indicators, the occurrence frequency of indicators and the availability of data in the network, the first round of screening of the index system is carried out, and 100 specific indicators are obtained to form the basic index system library. The index system library is the collected and referenced index system and the basis for the subsequent confirmation of the index system; Finally, by investigating the 
current situation of the research scope and cities of the same type, this paper makes a second round of screening on the index system library through collinearity test on the index data, and obtains 25 specific indicators, as shown in Figure 5.

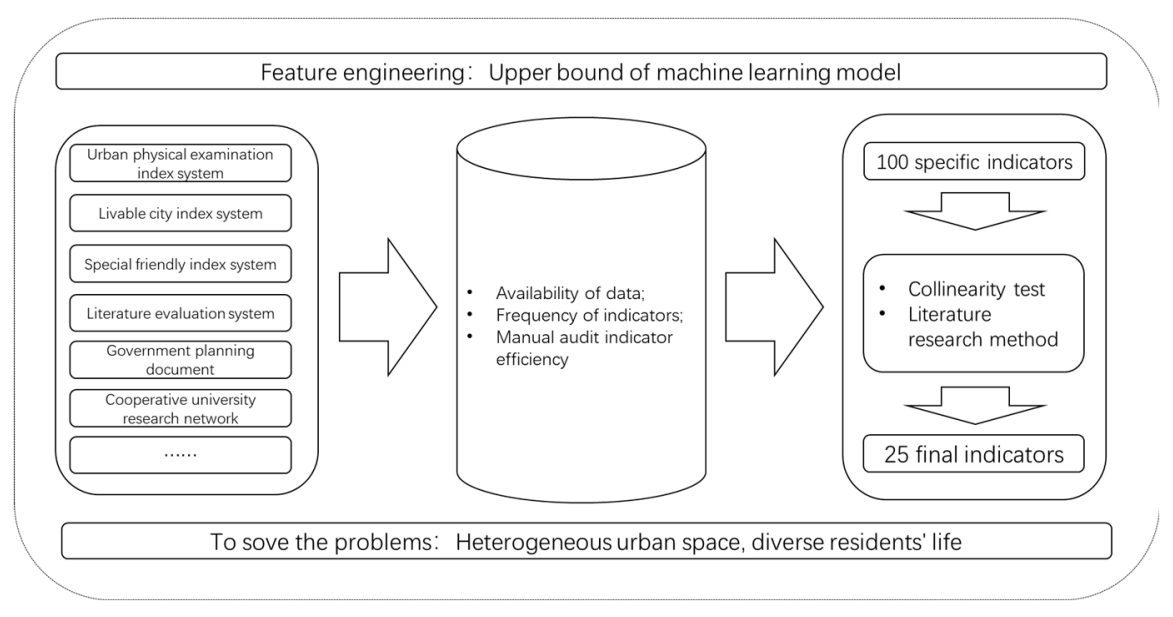

Figure 5 Acquisition process of evaluation index system

The data samples of index systematization are put into RF model training in different dimensions. The training results show that the model accuracy fluctuates between $0.75-0.92$, and the learning accuracy is high, which has been proved in previous studies ${ }^{28}$. This experiment analyses the whole age friendliness index system of basic communities in six districts and one county of Changsha, and identifies the key influencing factors in the process of community construction.

Through Grid-Search-CV search method and hyper-parameters sensitivity analysis, the RF weight of each evaluation index is obtained under the condition of optimal model accuracy, as shown in Table 2. (1) For the dimension of public friendliness, RF identified the park square accessibility, emergency shelter accessibility, medical facility accessibility and public security facility accessibility as the four most important indicators, with a total importance ratio of $44.50 \%$, while catering facility accessibility and subway station accessibility are considered to be the two least important indicators, with a total importance ratio of $5.60 \%$ It is found that all indicators in this dimension are relatively balanced, and the proportion of importance is normally distributed. (2) for the youth friendly dimension, it is found that the accessibility of community public service centres is the most important indicator, accounting for $32.50 \%$, which is far higher than other indicators; (3) For the child friendly dimension, the accessibility of primary schools and kindergartens rank the top two with a slight gap; from the elderly friendly dimension, the accessibility of entertainment facilities for the elderly and elderly care facilities are not much different; (4) Finally, based on the concept of age friendliness, on the whole, it is found that the public friendliness ranks first with $39.30 \%$, far exceeding other indicators, while other indicators fluctuate little, mostly around $19 \%$.

The above statistical analysis shows that in the process of community construction, residents' leisure, safety and health are the key influencing factors of public friendliness; at the same time, for the influencing factors in the youth stage, the accessibility of community public service centres is more important, while the demand for culture and entertainment accounts for a relatively small proportion; in contrast, the construction of child friendliness and elderly friendliness has a greater demand for cultural activities and entertainment In the whole age friendly community assessment, public friendliness, as the common demand space of the three age groups, represents the liveability of the community and the basic needs of development and construction. Therefore, under the comprehensive consideration of the whole life cycle development and individual growth, public friendliness is the most basic and important dimension, as shown in Figure 6. 
Luo, G.Q.;

Liang, C.; Huang, J.L.
Intelligent Assessment, Diagnosis and Planning

of All-age Friendly Communities Based on

Random Forest

Compared with other methods, RF can reflect the internal relationship between All-age friendly dimensions and index attributes, and express these relationships in the form of reduced value of Gini index, which is finally reflected as the difference between index weights, that is, the index weights of the two are hidden in the mapping relationship between evolution rules or network structure in the classification tree, which is more in line with the nonlinearity of age friendly concept At the same time, the code operation results show that RF has high accuracy and strong robustness of data mining, and can maintain the accuracy of $75 \%$ $\sim 92 \%$ in a small number of training samples, indicating that the Random Forest algorithm has good generalization ability and stability.

Table 2 Identification results of key factors of friendliness

\begin{tabular}{|c|c|c|c|}
\hline Dimension & Feature & Importance & Proportion \\
\hline \multirow{14}{*}{ Public Friendliness } & Accessibility of Parks & 0.133 & $13.30 \%$ \\
\hline & Accessibility of Emergency shelters & 0.118 & $11.80 \%$ \\
\hline & Accessibility of Medical facilities & 0.099 & $9.90 \%$ \\
\hline & Accessibility of public security facilities & 0.095 & $9.50 \%$ \\
\hline & Accessibility of parking facilities & 0.082 & $8.20 \%$ \\
\hline & Accessibility of Public toilet & 0.08 & $8.00 \%$ \\
\hline & Accessibility of sports and leisure facilities & 0.069 & $6.90 \%$ \\
\hline & Accessibility of living service facilities & 0.068 & $6.80 \%$ \\
\hline & Accessibility of waste dump & 0.059 & $5.90 \%$ \\
\hline & Accessibility of Shopping facility & 0.058 & $5.80 \%$ \\
\hline & Accessibility of Bus stop & 0.045 & $4.50 \%$ \\
\hline & Accessibility of fire fighting facilities & 0.038 & $3.80 \%$ \\
\hline & Accessibility of catering facilities & 0.03 & $3.00 \%$ \\
\hline & Accessibility of Subway station & 0.026 & $2.60 \%$ \\
\hline \multirow{5}{*}{ Youth Friendliness } & Accessibility of Community Public Service Center & 0.325 & $32.50 \%$ \\
\hline & Accessibility of sub district offices & 0.239 & $23.90 \%$ \\
\hline & Accessibility of cultural venues & 0.239 & $23.90 \%$ \\
\hline & Accessibility of Secondary school & 0.157 & $15.70 \%$ \\
\hline & Accessibility of Third space & 0.041 & $4.10 \%$ \\
\hline \multirow{3}{*}{ Child Friendliness } & Accessibility of Primary school & 0.353 & $35.30 \%$ \\
\hline & Accessibility of Kindergarten & 0.344 & $34.40 \%$ \\
\hline & Accessibility of child friendly facilities & 0.282 & $28.20 \%$ \\
\hline \multirow{2}{*}{ Elder Friendliness } & Accessibility of elderly leisure facilities & 0.512 & $51.20 \%$ \\
\hline & Accessibility of elderly care facilities & 0.488 & $48.80 \%$ \\
\hline \multirow{4}{*}{ All-age Friendliness } & Public Friendliness & 0.393 & $39.30 \%$ \\
\hline & Elder Friendliness & 0.223 & $22.30 \%$ \\
\hline & Child Friendliness & 0.194 & $19.40 \%$ \\
\hline & Youth Friendliness & 0.189 & $18.90 \%$ \\
\hline
\end{tabular}




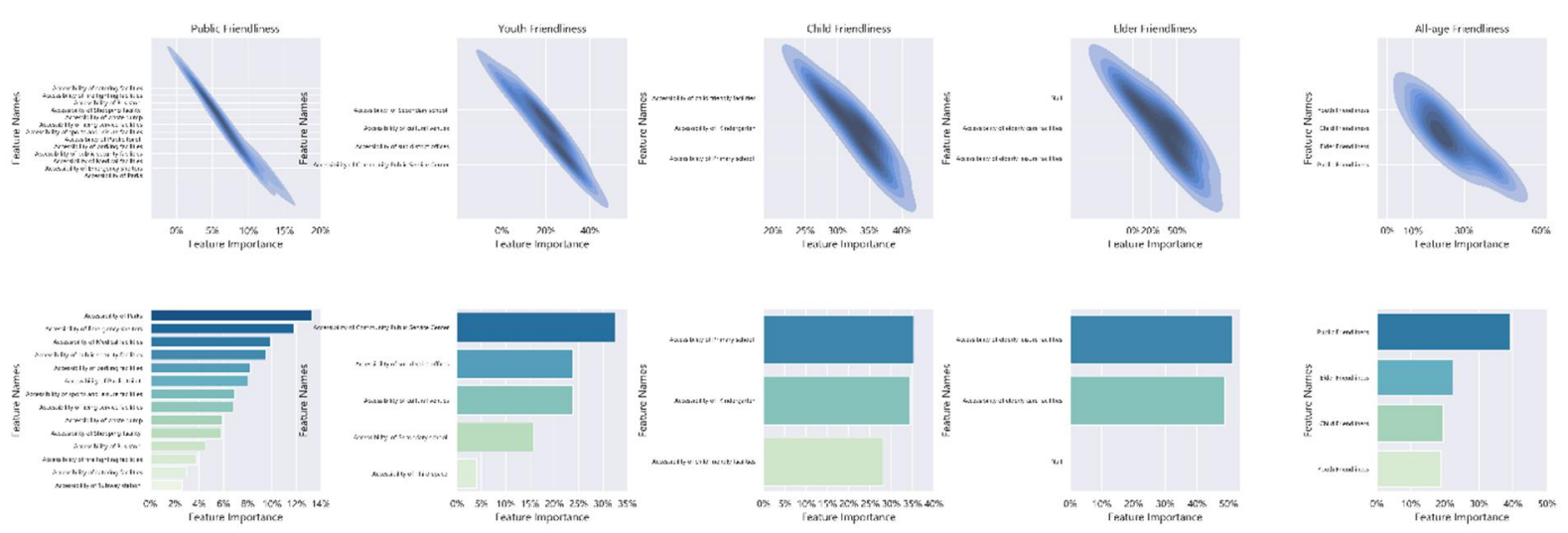

Figure 6 Statistical chart of characteristic importance and density of indicators in each dimension

\subsection{Problem diagnosis: Friendly diagnosis based on type classification}

The whole region is predicted by Random Forest algorithm, and the prediction results are divided into 1-5 levels, corresponding to very poor, poor, general, good and excellent respectively. The prediction results are displayed by ArcGIS (Figure 7) for spatial feature identification and analysis: (1) From the comparison of the spatial characteristics of individual friendliness of six districts and one county in Changsha, the community friendliness in Changsha has little difference, and all friendliness presents a trend In the "coreedge" pattern, communities with high friendliness are mostly concentrated in Eastern site of Changsha (especially Yuhua District), indicating that there is a strong correlation between the current situation of community friendliness and the development degree of community and surrounding environment; (2) From the spatial distribution characteristics of individual friendliness, the distribution of high score areas in the six districts of Changsha is relatively concentrated. Based on the flow of people, the agglomeration state of commercial space and the relationship ${ }^{29,30}$ between residential space in Changsha, the current situation of community construction in the central urban area of Changsha and the complete commercial space pattern (commercial central area, urban commercial belt and specialized commercial area) The development status is similar, with the community construction around Wuyi business district, Dongtang business district and Desiqin business district as the representative; (3) From the analysis of the prediction index results of individual friendliness, the community friendliness of Changsha shows aggregation at the extreme value as a whole, in which the communities in extreme state account for about $37.89 \%$ and $36.34 \%$ respectively, but the friendliness of the elderly and children is high Figure 8.

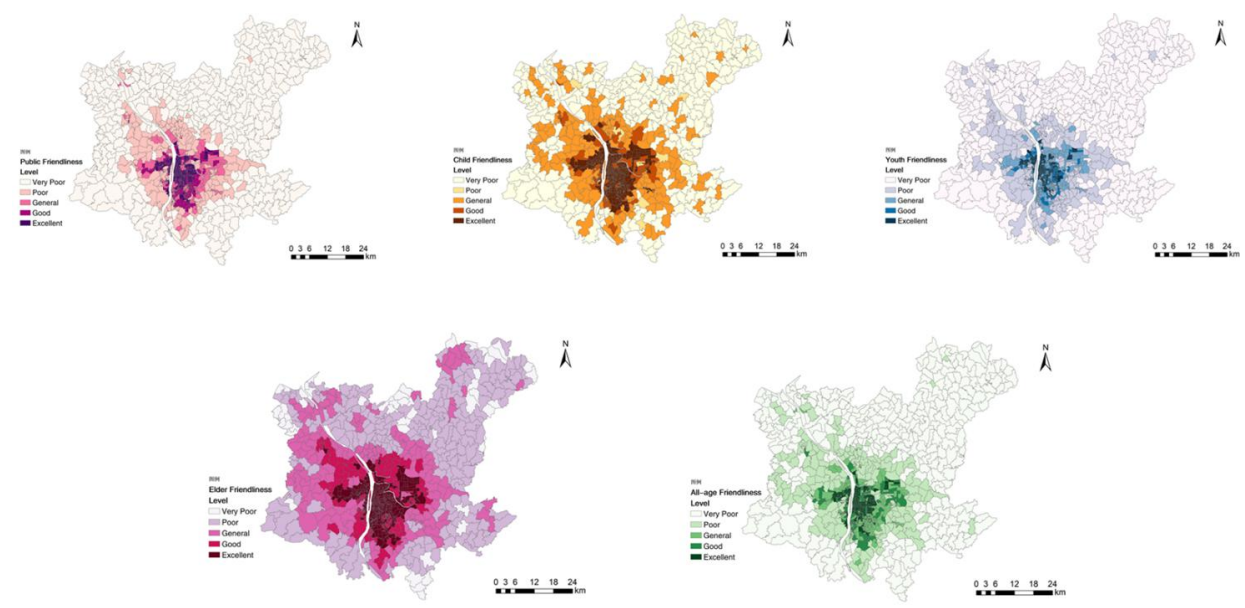

Figure 7 Spatial distribution map of community friendliness evaluation of six districts and one county in Changsha 

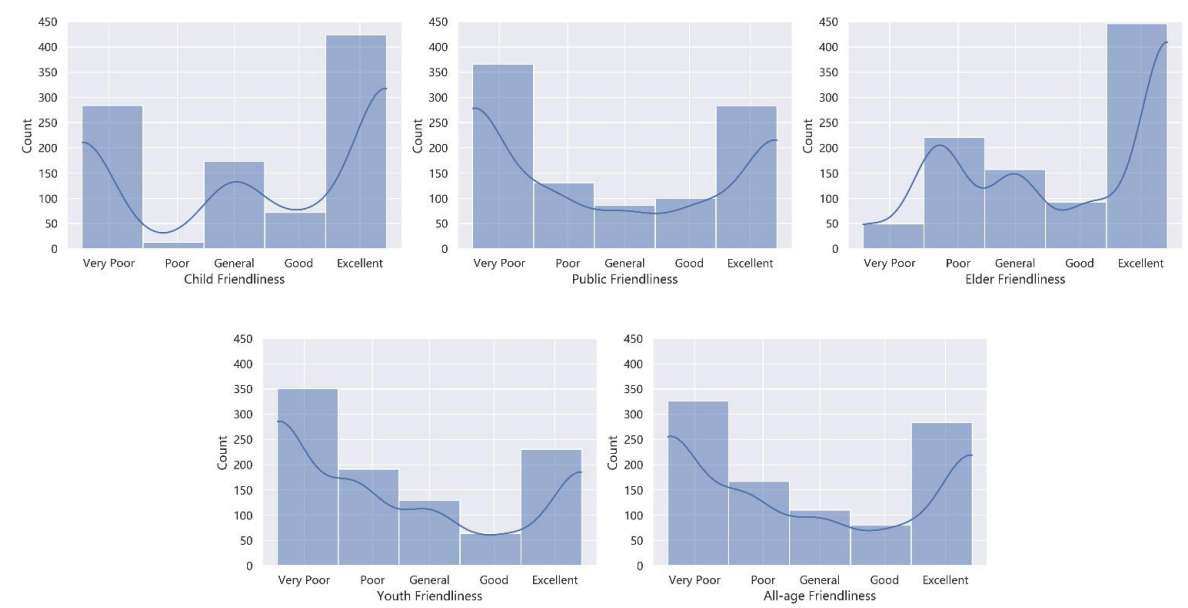

Figure 8 Statistical map of community friendliness evaluation of six districts and one county in Changsha

\subsection{Planning solution: Planning governance response based on friendly diagnosis}

Based on the results of the existing projects such as liveable community, child friendly community and urban physical examination in Changsha and the experimental evaluation results of community friendliness within six districts and one county in Changsha, taking public friendliness as the bottom line standard, child friendliness as the special goal and All-age friendliness as the ultimate goal, a total of three typical communities were selected for public friendliness, child friendliness, youth friendliness Carry out case analysis on the elderly friendly and all age friendly construction( Figure 9), and realize the whole process of case community assessment, diagnosis and planning on the basis of assessment.

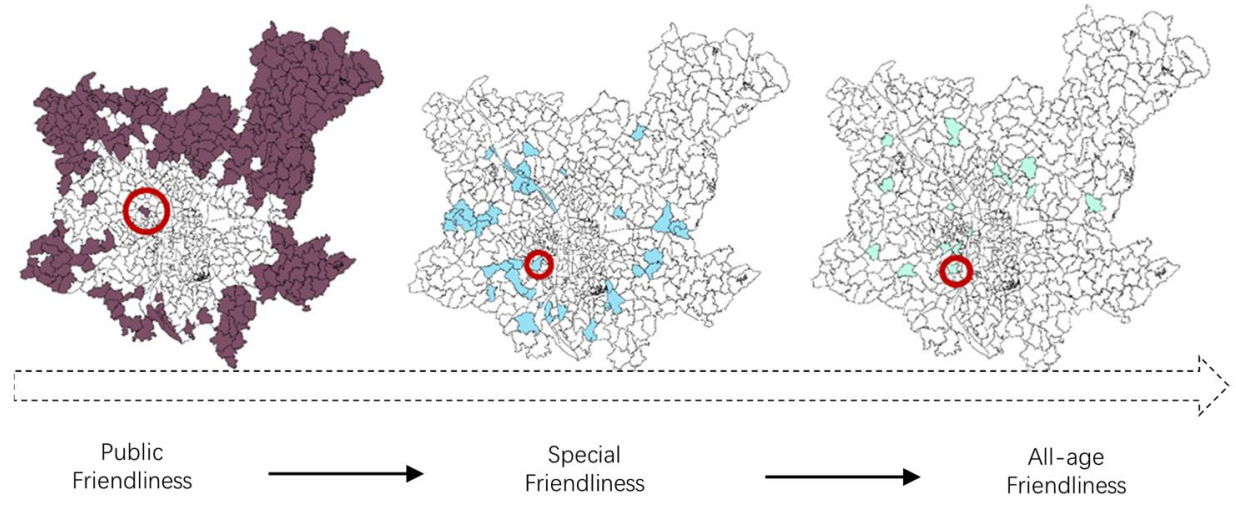

Figure 9 Flow chart of whole age friendly community construction

\subsubsection{Public-friendly oriented community}

Taking cs-01 community as an example, based on the multi-dimensional perspective of full-age friends, it is found that the community has a weak degree of public friendliness, and the in-depth special friendly construction can only be carried out after the construction of public friendly facilities and the improvement of indicators.

Through the specific calculation of bucket theory ${ }^{31}$, the types of medical facilities to be optimized under the goal of public friendliness in the community and the specific short board spatial distribution of the unit community are obtained. The specific performance is that the accessibility of medical facilities within the community is insufficient, and the short board area is mainly concentrated in the centre of the unit community (as shown in Figure 10-A). Based on the community evaluation results and short board 
diagnosis results, the community planning project selection is obtained after benchmarking with the existing project case base. The planning project is intelligently selected through multi-agent calculation. By making the decision table, according to the citizen demand level agent (as shown in Figure 10-B), people flow agent (as shown in Figure 10-D) and government agent, it is observed that the short board core area of cs-01 community is distributed in the middle of the site, while the population distribution shows the characteristics of high in the South and low in the north. Based on the decision results of multiple agents, the belt-shaped site selection recommendation area in the middle of the site is obtained (Figure 10-e).

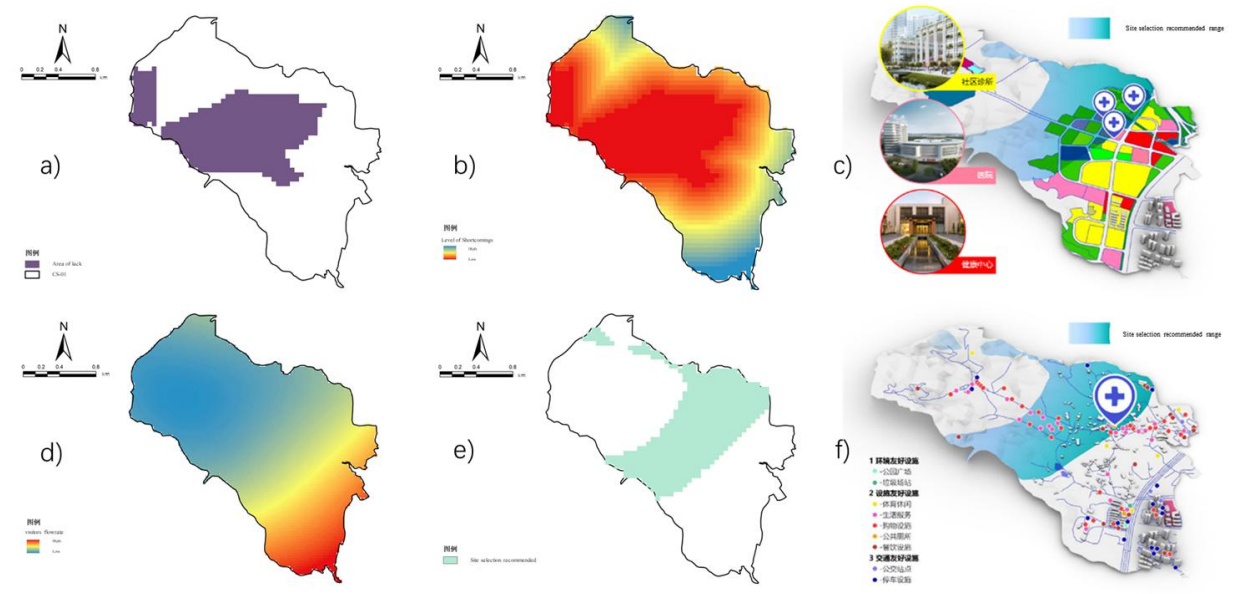

Figure 10 CS-01 community analysis result

By comparing the control plan with the current $\mathrm{POI}$ and visitors flowrate data analysis, it is found that a piece of medical facility land is planned in the southeast of the original control plan, but it has not been implemented in practice, and the change of population flow also reflects the insufficient consideration of the original control plan. It is suggested that the living service facilities should be concentrated, and there should be a commercial community in the south, a primary school and a kindergarten respectively. The proposed site is located in the residential area near the scenic spot (as shown in Figure 10-f). Then combine the original scheme with the current situation of the community, re invest in the evaluation system for systematic evaluation, and get that the original planning scheme still needs to be optimized. In the optimization scheme, it is suggested to divide the future planning of the unit into residential, commercial and school comprehensive plates, and add medical facilities in combination with the nature of the land to form the project planning intention. In this experiment, RF model, barrel theory and multi-agent method are integrated to accurately identify the shortcomings of medical facilities in the community, which provides scientific calculation, identification and support for the adjustment and comparison of planning schemes.

\subsubsection{Special-friendly oriented community}

Taking CS-02 community (as shown in Figure 11) as an example, according to the evaluation of community construction friendliness, the community has reached the public basic friendliness index, but there is a lack of special friendliness construction. The whole age friendliness construction can only be carried out after the construction of special friendliness facilities and the improvement of indicators. Through RF model calculation, the optimal type of educational facilities for child friendly goals in the community is obtained.

According to the barrel theory calculation, the diagnosis result of the short board in the case unit is that there is a short board in the primary school, which is specifically reflected in the lack of accessibility of the primary school in the community within six districts and one county, and the space short board covers almost the whole community unit (Figure 11-a). According to the short board level (citizen demand level agent), passenger flow agent and government agent, and the spatial distribution characteristics of 
population (Figure 11-d), the proposed site selection range on the east side of the site is comprehensively obtained (Figure 11-f).

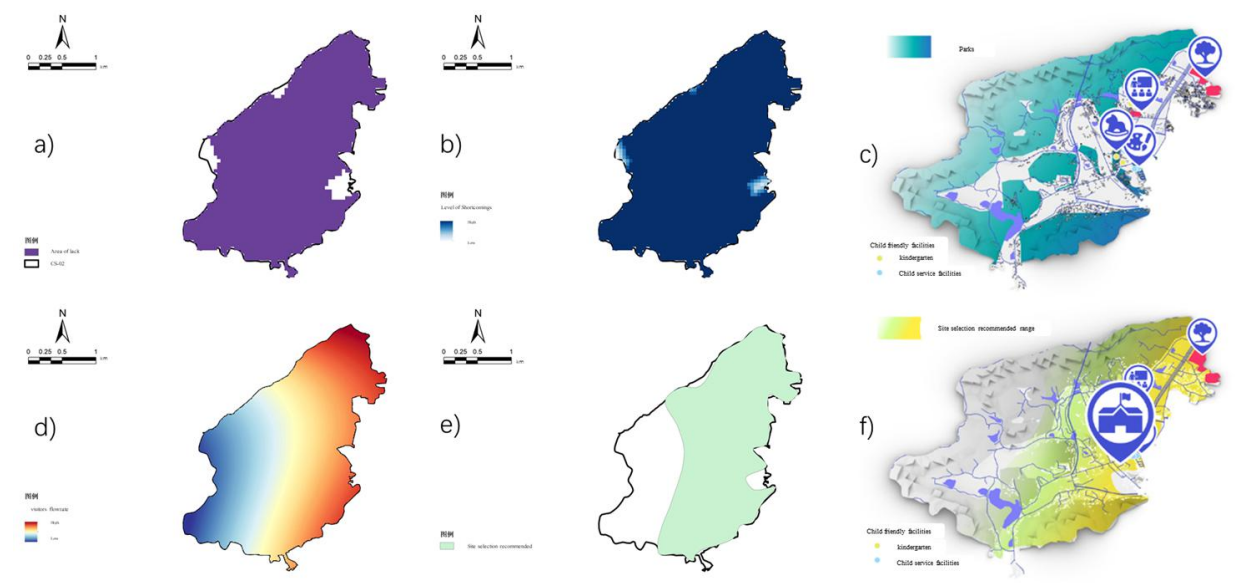

Figure 11 CS-02 community analysis result

Through the calculation and analysis of the whole technology system model, it is recognized that there is a lack of development power of the child friendly dimension in the community, which is specifically manifested in the lack of child friendly facilities such as primary schools. The imbalance between supply and demand leads to social contradictions in the community. Through quantitative model identification, find out the causes, put forward planning suggestions, and get the suggestions on the optimization scheme of multi-agent location decision (Figure 11-f). The proposed location area of primary school is located in the east of the site close to the Ring Road area. Combine the distribution status of child friendly facilities and available space to form the intention of primary school project placement.

\subsubsection{Age-friendly oriented community}

Taking CS-03 community as an example, according to the evaluation of community construction friendliness of Random Forest model, the community has achieved the objectives of basic friendliness and special friendliness, but there is still a lack of full-age friendliness. The specific evaluation results are youth friendly target house prices to be optimized.

According to the calculation of Random Forest model and barrel theory, there is a youth friendly short board in the community unit, which is caused by the high level of house price. The short plates are concentrated on the southeast side of the unit (Figure 12-a). According to the public demand level agent, traffic agent and government agent, and the characteristics of population distribution, the northeast and southwest corners are relatively high, and the recommended range of house price optimization is obtained comprehensively (Figure 12-e).

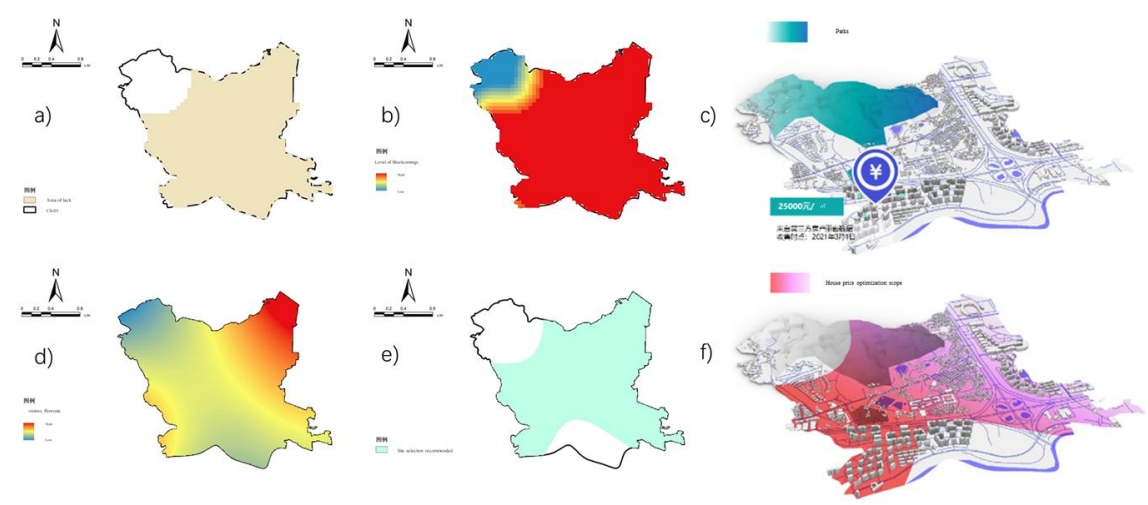

Figure 12 CS-02 community analysis result 
Through the model calculation, it is found that the biggest problem of this unit is the house price level under the youth friendly dimension. Looking at the house price level in this area, it is found that the new house price in the high-end residential area in the south is as high as $25000 \mathrm{RMB} / \mathrm{m}^{2}$ (the average price in Changsha is $10200 \mathrm{RMB} / \mathrm{m}^{2}$ ), which is significantly higher than the surrounding house price level. Under the age friendly goal, house price factors significantly affect the friendliness to young people (Figure 12-c). Therefore, it is suggested to optimize the scheme (Figure 12-f). House prices can be controlled through policy regulation and other behaviours. In this area, attention should be paid to the demand scenarios of young talents for work, life and leisure nearby. Form a dynamic, diversified and inclusive innovation and development functional plate. It is suggested to give priority to pilot diversified housing forms, encourage both rental and sales, and control the healthy and stable development of housing prices in the sector. Thus, the model can not only find out the problem of insufficient supply of facilities, but also find the lack of policy regulation.

\section{Conclusion and discussion}

\subsection{External characteristics and internal mechanism of spatial differentiation in all age friendly communities}

Under the guidance of people-oriented planning concept, this study focuses on the multi-dimensional subject status evaluation and planning intervention of full-age friendly communities, and constructs quantitative analysis and evaluation methods such as the full-age friendly community evaluation system model based on Random Forest (RF), so as to accurately evaluate the multi-dimensional friendliness in community construction; On the basis of "public friendliness", this paper introduces the humanistic care concept of "child friendliness, youth friendliness and elder friendliness", explores the community construction mechanism, analyses the coupling between multi-dimensional factors and multiple subjects under the concept of "all age friendliness" community planning, constructs a scientific and effective theoretical index system, and interprets the construction mechanism of "all age friendliness"; And constantly strengthen the interactive relationship between the theoretical system and the current situation, optimize the quantitative analysis model, and improve the community evaluation methods and construction approaches based on the whole life cycle theory.

External characteristics: the research shows that at the community planning level, this research practice is based on the evaluation results of each stage, takes "public friendly supplement - child friendly promotion - all age friendly building" as the special evaluation goal of this practice, and selects a case for research and analysis of the community units to be improved identified at each stage. From a global perspective, on the premise of high model accuracy, the friendly values in the five dimensions show the spatial distribution characteristics of "core edge". The high value range is mainly concentrated in the part, east area of the river, of the central urban area, and gradually decreases from the traffic trunk line to the periphery; From a local perspective, child friendliness and old age friendliness cover a wider range than other dimensions.

Internal mechanism ${ }^{32}$ : the rationalization of government decision-making is the influencing factor of the construction of age-friendly community. In recent years, Changsha has been trying to build a friendly city. Actions such as "child friendly city" and "elderly friendly community" emerge one after another, such as Furong district, and the concept of building a age-friendly city is becoming stronger and stronger; Road traffic is the development link of age-friendly. As the core area of age-friendly community in Changsha, Furong district is also the economic centre of Changsha. It plays a radiating role to the surrounding communities according to the surrounding roads and leads the development of the community.

\subsection{Significance of Random Forest Algorithm to Governance Practice}


The research introduces the concept of "All-age friendliness" to measure the level of community construction from the needs of residents in the whole life cycle, which has certain practical significance and embodies the concept of "human-oriented". At the same time, based on the targeted short board of the community and the current situation evaluation results, the model puts forward the targeted community planning scheme, and provides suggestions on the landing scope for the planning scheme. From the theoretical and practical aspects, the model realizes: (1) The coupling of multi-source data system and multi-dimensional index system. Through the characteristic engineering structure of machine learning, scientifically evaluate the current situation of community construction from a multi-dimensional perspective, analyse and excavate the complex relationship between multi-dimensional influencing factors and multi-stage subjects, and construct the cognition of the integrity of community life cycle construction; (2) Shortcomings of community construction is coupled with the key factors of the community. Through the bucket theory and Random Forest algorithm, it can accurately identify the deficiencies of community construction and key elements of development, deeply tap the internal potential of the development process of full-age communities, and interpret the basis for community construction evaluation and development decision-making; (3) Innovative coupling between community planning scheme and governance implementation. Through multi-agent decision-making location, after the planning mode scheme matching is completed, the comprehensive evaluation spatial visualization results are introduced to comprehensively identify the location scope of the planning and governance scheme and improve the realizability of the planning scheme.

\subsection{Discussion}

Based on the Random Forest algorithm and the concept of the whole life cycle, this paper studies the community-scale age-friendly evaluation, diagnosis and planning methods in Changsha, and comprehensively considers the public space needs of the whole life cycle and the specific needs of each age group. The index system is divided into four dimensions and specific categories: public friendliness, youth friendliness, child friendliness and elderly friendliness, and it provides a certain basis for the diagnosis and planning of pain points in the process of follow-up community development, and can be tested through cases. But at the same time, there are still many deficiencies in the research: (1) the rationality and effectiveness of the index system need to be strengthened. Based on the existing research results and data acquisition, the index system listed has a certain regionality, which is not universal in regions or cities with different degrees of development. At the same time, the current research only focuses on space, and only objective indicators such as spatial accessibility can be used to replace the economic income, living preference, living habits, travel intention and other data of residents in different areas; (2) This research still stays at the spatial two-dimensional level. The Random Forest algorithm can adjust the research accuracy and the importance of key influencing factors according to the real-time updated sample data, but the comprehensive consideration of time and space is insufficient at the index level, and the adaptability to the development gradualness, mining and prediction pattern formation mechanism and evolution trend still needs to be further studied; (3) Training samples are screened and matched by the liveability research results. There are some errors in the matching method, which needs to be optimized. Due to some differences in research ideas and footholds, there are some deviations in the process of matching the training samples with the data calculation results of the index system, which may have a certain impact on the experimental results; (4) Integration of evaluation results and planning innovation needs to be optimized. The research mainly focuses on Method Exploration and evaluation innovation. At the same time, community planning has pertinence and specificity. The research on Intelligent innovation of specific community planning scheme should be further deepened according to the specific development level of the community and the real needs of residents. 


\section{References}

\footnotetext{
${ }^{1}$ Beijing Municipal People's Government.(2005) Beijing city's overall planning (2004-2020).

2 Li X.Y. (2019) A Review on the Research Progress of Age-friendly Communities Abroad, [J]. Urban Development Studies, 2019,26(07):14-19.

${ }^{3} \mathrm{Ma}$ C.P. (2019). Application Research on Optimization Strategy of "full age" community elderly care system (Master's thesis, Changchun Institute of Engineering)

https://kns.cnki.net/KCMS/detail/detail.aspx?dbname=CMFD201901\&amp;filename=1019021214.nh

${ }^{4}$ Liu Z.C. (2014). Research on space design of child friendly community (Master's thesis, Southwest

Jiaotong University)

https://kns.cnki.net/KCMS/detail/detail.aspx?dbname=CMFD201601\&amp;filename=1015348933.nh

${ }^{5}$ Huang J.L., Li Z.Y., etc. (2019) Child Friendly Planning Practice towards Communicative Action, Changsha, [J]. Planners, 2019,35(01):77-81+84.
}

${ }^{6}$ Zhu F., Zhang J.Q., etc. (2019) The Reasons for the Rise, the Evaluation Strategies and the Enlightenment for Youth-friendly Cities in Developed Countries, [J]. Youth Research, 2019(02):71-80.

${ }^{7}$ Zhu F. (2018) Research on Innovative Competition of the Policy for "New First Tier Cities" Youth Friendly Cities, [J]. China Youth Study, 2018(06):78-85.

${ }^{8}$ Zhou D., Xu Y.S. (2014) Planning and Indicators Control of Urban Residential Space in An Aging Society, [J]. Architectural Journal, 2014(5), 56-59.

${ }^{9}$ Wu P.Q., Zhang Y.Y., etc. (2016) Research on all-age community site selection based on GIS--A Case study of Fuzhou downtown zone, [J]. Journal of Fuzhou University(Natural Science Edition),

2016,44(5):668-679.

${ }^{10}$ Liu T.Q , Zong K. (2012) On the planning and design of comprehensive elderly care community--A case of Guoshou Langfang ecological health city, [J]. Construction \& Design for Engineering, 2012(11): 815.

${ }^{11}$ Li X.Y. (2019) Review on the research progress of elderly friendly communities abroad, [J]. Urban development research, 2019,26 (07): 14-19

$12 \mathrm{Ma}$ C.P. (2019). Application Research on Optimization Strategy of "full age" community elderly care system (Master's thesis, Changchun Institute of Engineering)

https://kns.cnki.net/KCMS/detail/detail.aspx?dbname=CMFD201901\&amp;filename=1019021214.nh

${ }^{13}$ Liu L.W., Shen L.J., etc. (2016) Senior Community Planning For All Ages, [J]. Planners, 2016,32(10):99102.

${ }^{14}$ All-Age-Cities Final-Version-Keri-August-7th[R], Canada, 2014.

${ }^{15}$ Xie B., Wei W., etc. (2015) Senior Community Space Evaluation And Planning, [J]. Planners, 2015,31(11):

$5-11$.

${ }^{16}$ Zhang L., Ye Z. (2020) All-age Regeneration Strategy of Existing Residential Areas Based on the Compound Feature of the Aged and the Young, [J]. Urban Development Studies, 2020, 27(10):109$115+133$.

${ }^{17}$ Zhao D.X., Sun J.L.. Study on evaluation index system of livable environment for urban elderly in China[J]. Environmental Protection and Circular Economy, 2013,33(07):52-55.

${ }^{18}$ Zhang W.Z. (2007) Index System and Method of Residential Environmental Evaluation in Inner Cities, [J]. Scientia Geographica Sinica, 2007(01): 17-23.

${ }^{19}$ Meng X. (2020) Planning practices and implications on the overseas Child Friendly Cities, [J]. Urban Problems, 2020(03): 95-103.

${ }^{20}$ Zhou J., Huang J.L., Zeng Y.J., Duan X. (2019). Construction and Empirical Study of community environmental livability evaluation model -- Taking 719 communities in Changsha metropolitan area as an example. China Urban Planning Society. (eds.) vibrant urban and rural beautiful habitat -- Proceedings of 2019 China urban planning annual conference (20 housing and community planning) (pp. 97-110). China Construction Industry Press

${ }^{21}$ Zhu Z.J. (2021) Friendly and inclusive create the city of a loved life for residents., [ J ] Pioneer, 2021 $(02): 42-44$.

${ }^{22}$ Zhou J. (2018) The evaluation of community environmental livability based on multi-source data, $[D$ ]. 
Shenzhen University, 2018.

${ }^{23} \mathrm{Nie}$ J.K., (2019) Research on the evaluation index system of livable communities oriented by residents ' daily living needs, [ J ].Modern urban research, 2019 ( 11 ) : 120-124.

${ }^{24}$ LEO B. (2001) Random forests. Machine Learning, 2001,45(1):5-32.

${ }^{25}$ Lai C G, Chen X H, etc. A flood risk assessment model based on Random Forest and its application[J]. Journal of Hydraulic Engineering, 2015, 46(01):58-66.

${ }^{26}$ LINDNER C, BROMILEY P A, IONITA M C, et al.(2015) Robust and accurate shape model matching using Random Forest Regression-Voting. IEEE Transactions on Pattern Analysis and Machine Intelligence, 2015, 37 (9) : 1862-1874.

${ }^{27}$ Hu H.L., Zeng Y.N., Zhang H.H., etc.(2011) Intergration of a Site Selection Model with the Multi-Agent System and the Colony Algorithm and Its Application to Changsha, [J]. Resources Science, 2011, 33(06):1211-1217.

${ }^{28}$ Chen D.L., Lu X.H., etc.(2019) Measurement of cultivated land utilization efficiency: Construction and application of random forest, [J]. Journal of Natural Resources, 2019,34(06):1331-1344.

${ }^{29}$ Ye Q., Zhao Y., etc.(2021) Research on Retail Commercial Space Agglomeration and Its Influencing Mechanism Based on Spatial Heterogeneity: A Case Study of Changsha, [J]. Modern Urban Research, 2021(01):52-58.

${ }^{30}$ Ye Q., Tan Y.T., etc. (2011) The impact of large-scal shopping centers on urban commercial spatial structure--A Case of Changsha city, [J]. Economic Geography, 2011, 31(03):426-431.

${ }^{31}$ Zhong T., Wu H.F., etc. (2020) Construction of Regional Water Supply Safety Index System and Identification of Short Board Elements, [J] Water Resources and Power, 2020,38(02):52-55.

${ }^{32}$ Fang F., He R.W. (2018) Study on the spatial evolution characteristics and mechanism of Rural producing, living and ecology from the perspective of farmers' behavior, [J] Study and Practice, 2018(01):101-110. 